\title{
O PAPEL DOS MINICURSOS NA CAPACITAÇÃO DE ALUNOS PARA UTILIZAÇÃO DE FERRAMENTAS NECESSÁRIAS EM DISCIPLINAS DO CURSO DE ENGENHARIA ELÉTRICA: EXPERIÊNCIA DO PET
}

Anatelli A. F. Heringer - anatellih@gmail.com

Larissa B. Grassi - larissabg50@gmail.com

Matheus B. Luchini - matheusbluchini@gmail.com

Murilo S. de Mendonça - murilosantolini@gmail.com

Paulo J. M. Menegáz - paulo.menegaz@ufes.br

Universidade Federal do Espírito Santo

Av. Fernando Ferrari, 514 - Goiabeiras

29075-910 - Vitória - Espírito Santo

Resumo: A Engenharia Elétrica é uma vasta área do conhecimento, não sendo possível a exploração, nos cursos de graduação, de todos os seus campos de atuação e ferramentas necessárias na compreensão dessas. Os minicursos ofertados pelo PET Engenharia Elétrica Ufes se mostram, diante desse cenário, como uma alternativa para a complementação do exposto em sala de aula, bem como das exigências do mercado de trabalho. Foi mostrado, portanto, por meios dos dados de participação e feedback dos minicursos aplicados, além de relatos dos autores (egressos do grupo), que o impacto dessa atividade é benéfico aos alunos e também ao curso em si. Também é detalhado que o desenvolvimento de atividades desse tipo (ministrar minicursos) está em perfeito alinhamento com os princípios do PET.

Palavras-chave: Minicursos. Engenharia Elétrica. PET Elétrica Ufes.

\section{INTRODUÇÃO}

\subsection{Motivação}

Atualmente, tem-se notado um mercado cada dia mais exigente e competitivo, buscando profissionais que apresentam constante atualização e capacitação em suas respectivas áreas. Com isso, torna-se necessário o preparo para os desafios constantes e com respostas rápidas às necessidades das organizações.

As instituições de trabalho buscam por profissionais com habilidades técnicas, e também habilidades humanas e conceituais. Eles têm que estar alinhados aos objetivos estratégicos da organização em que trabalham. A partir disso, torna-se cada dia mais imprescindível um maior preparo por parte do aluno de graduação, buscando conhecimentos fora da sala de aula tradicional, de forma a complementar sua formação. Nesse contexto, projetos extracurriculares como Iniciações Científicas, Projetos de Extensão, cursos em parcerias com empresas ou ofertados por programas de educação da própria instituição de ensino a respeito de ferramentas específicas se tornam grandes auxiliares nessa jornada do futuro profissional. 


\subsection{O Programa de Educação Tutorial - PET}

Em 1979, foi criado o Programa Especial de Treinamento - PET pela Coordenação de Aperfeiçoamento de Pessoal de Nível Superior - CAPES. Em 2004 o PET passou a ser identificado como Programa de Educação Tutorial. Em 23 de setembro de 2005 foi regulamentado pela Lei $\mathrm{N}^{\circ} 11.180$ e, em 29 de setembro de 2005 pela Portaria MEC $\mathrm{N}^{\circ}$ 3.385. O PET é um programa do Governo Federal brasileiro, estando, a partir de 2000, subordinado à Secretaria de Ensino Superior (SESu) do Ministério da Educação (MEC) (BRASIL, 2013, pg. 14).

O Programa é composto por um professor tutor e alunos de graduação, sejam eles bolsistas ou voluntários, que realizam atividades extracurriculares, baseadas no tripé acadêmico (pesquisa, ensino e extensão) e, assim, complementam a sua formação acadêmica, procurando atender às necessidades do próprio curso de graduação e aprimorar os objetivos e conteúdos programáticos da grade curricular, além de estimular valores que reforçam a cidadania e a consciência social dos participantes (BRASIL, 2010, pg. 40).

O PET é um programa vinculado institucionalmente à Pró-Reitoria de Graduação e atualmente conta com 842 grupos distribuídos entre 121 IES (MEC, 2018). Na Universidade Federal do Espírito Santo (Ufes) temos 13 grupos PET: Administração, Conexões Cultura, Economia, Educação Física, Engenharia de Computação, Engenharia Elétrica, Engenharia Mecânica, Conexões Educação, Conexões Licenciatura, Matemática, Psicologia e Serviço Social no campus de Goiabeiras e o PET Conexões ProdBio no campus de São Mateus (PET Ufes, 2020). O grupo PET Engenharia Elétrica Ufes nasceu em 2006 e contou com um total de 4 tutores, centenas de alunos e inúmeros projetos, desde sua criação.

\subsection{O Curso de Graduação em Engenharia Elétrica}

O curso de Engenharia Elétrica da Universidade Federal do Espírito Santo (Ufes) foi criado em 1971. Atualmente, ele está sediado no Centro Tecnológico do Campus de Goiabeiras, na capital Vitória. Desde 2017, o ingresso se dá por meio do SISU com duas ofertas anuais de 40 vagas em cada semestre (DEE/UFES, 2020).

Com duração de 5 anos, a última alteração curricular, realizada em 2009, definiu a carga horária mínima em 4.215 horas dentre disciplinas obrigatórias, optativas, estágio, atividades complementares e Projeto de Graduação, dando aos graduandos a possibilidade de concluir o curso com uma ou mais das seguintes ênfases: Computação, Controle e Automação, Eletrônica, Sistemas de Energia e Telecomunicações. O Departamento de Engenharia Elétrica é composto por 37 professores, dos quais 34 são doutores, 1 é mestre e 2 são engenheiros (DEE/UFES, 2020).

O curso teve, ao longo de sua história, papel fundamental na formação de engenheiros eletricistas no Espírito Santo, tendo sido, por muitos anos, a única opção gratuita disponível para a população, pois apenas em 2006 o Instituto Federal do Espírito Santo passou a oferecer o curso de Engenharia Elétrica no campus de Vitória (MEC, 2020). O curso da Ufes é responsável pela formação de 1646 engenheiros eletricistas, desde sua criação.

Em termos avaliativos, o curso tem sua qualidade amplamente reconhecida, possuindo, atualmente, Conceito 5 no Enade e tendo conquistado a quarta maior nota do país na edição de 2017 do exame. No Ranking Universitário Folha encontra-se atualmente na $20^{a}$ posição nacional (Folha de S. Paulo, 2020). Além do curso de graduação, a Ufes também oferece cursos de Pós-Graduação em Engenharia Elétrica, mestrado e doutorado, ambos com 
Conceito Capes 5 (CAPES, 2020), tendo formado mais de 400 mestres e 140 doutores (Engenharia Elétrica Ufes, 2020).

\section{A IMPORTÃNCIA DO ENSINO DE FERRAMENTAS AUXILIARES PARA USO EM DISCIPLINAS DA GRADE CURRICULAR}

As disciplinas necessárias à integralização curricular são responsáveis por 3.360 horas da carga horária mínima do curso. Elas são ofertadas, em sua maioria, pelo departamento de Engenharia Elétrica (70\%), além dos departamentos de: Informática, Matemática, Física, Química, Estatística, Engenharia Mecânica, Engenharia de Produção e Engenharia Ambiental (UFES, 2009).

Em várias dessas disciplinas, é indispensável a utilização de ferramentas externas à disciplina para efetivação do conhecimento por meio da realização de simulações, trabalhos, avaliações, etc. Em geral, tratam-se de ferramentas computacionais, tais como: Matlab para simulação em disciplinas das mais diversas áreas (telecomunicações, controle e automação, sistemas de potência); AutoCAD e DIALux para elaboração de projetos elétricos; pacote Office, com aplicações diversas; Latex para elaboração de documentos (relatórios, artigos, Projeto de Graduação); Arduino, aplicado na elaboração de projetos que envolvam eletrônica e automação; Eagle, para confecção e montagem de placas de circuito impresso, dentre outras.

A limitação de carga horária das disciplinas não permite, no entanto, que a orientação no que diz respeito à utilização dessas ferramentas seja feito de maneira efetiva. Resta aos alunos, então, buscar esse conhecimento fora da sala de aula a fim de poder dominá-las antes ou durante sua aplicação. Daí surge a extrema importância dos minicursos oferecidos por grupos que apoiam a graduação, dentre os quais destaca-se o PET, foco deste artigo.

\subsection{Aplicações das Ferramentas}

\section{Arduino}

O Arduino é uma plataforma eletrônica composta por um microcontrolador, circuitos de entrada/saída e que pode ser facilmente conectada à um computador e programada via IDE (Integrated Development Environment, ou Ambiente de Desenvolvimento Integrado) utilizando uma linguagem baseada em $\mathrm{C} / \mathrm{C}++$, apenas com o uso de um cabo USB. Depois de programado, o microcontrolador pode ser usado de forma independente, ou seja, pode ser colocado para controlar um robô, as luzes da uma casa, a temperatura do ar condicionado ou qualquer outro aparelho de medição, de forma a medir variáveis por sensores e controlar os atuadores para alcançar o objetivo requerido. Para isso, o Arduino possui uma quantidade enorme de sensores e componentes. Grande parte desse material está disponível em módulos, que são pequenas placas que contém os sensores e outros componentes auxiliares ou nos chamados Shields, que são placas que podem ser encaixadas no Arduino para expandir suas funcionalidades.

A vantagem do Arduino no meio acadêmico é o fato de ser um dispositivo que é, ao mesmo tempo, barato, funcional e fácil de programar, sendo dessa forma acessível a estudantes e projetistas amadores.

No curso, ele é utilizado principalmente em disciplinas práticas, como Práticas de Laboratório, Circuitos Elétricos, Eletrônica Básica, Laboratório de Controle Automático, Projeto Orientado, ou aquelas em que o professor exige um projeto final para exercitar 
habilidades práticas ou comprovar alguns critérios estudados em sala de aula, como por exemplo, Eletromagnetismo, Princípios de Comunicações e Sistema de Telecomunicações.

\section{Excel}

O Excel é uma ferramenta desenvolvida pela Microsoft que faz parte do Pacote Office, um conjunto de programas quase hegemônico nas esferas acadêmica e empresarial. Apesar da relativa facilidade de usá-lo para geração de tabelas simples e no gerenciamento de planilhas, seus recursos vão muito além disso, oferecendo desde variações de formatação e fórmulas lógico-matemáticas até uso de macros e programação em VBA. Esses últimos são recursos constantemente buscados quando o objetivo é rapidez e eficiência, sendo de grande utilidade para otimizar processos e automatizar tarefas corriqueiras.

No curso, ele é utilizado em diversas disciplinas nas mais diversas aplicações. Pode-se citar algumas de fundamental importância, como: no auxílio à elaboração de projetos elétricos em Instalações Elétricas I e II; em Gestão e Eficiência Energética, na realização de uma auditoria energética em uma Unidade Consumidora (avaliação dos diversos usos finais da energia elétrica com vistas à otimização). Sua versatilidade em gerar tabelas e gráficos também faz com que seja um software utilizado constantemente pelos alunos em outras disciplinas, como, por exemplo, Física Experimental, onde são usadas aproximações de curvas para embasamento teórico de experimentos práticos.

No caso dessa ferramenta, é de fundamental importância citar o valor em se dominar sua utilização quando se consideram os estágios oferecidos na área, uma vez que tal conhecimento é obrigatório ou então um grande diferencial no processo seletivo da maioria das vagas.

\section{Latex}

O Latex é um sistema para preparação de documentos, utilizado principalmente na área acadêmica, notadamente em Matemática, Física, Ciência da Computação e Engenharias. Ele é uma plataforma na qual se digitam textos com indicações de layout já definidas em linhas de comando pré-fixadas para posterior compilação e, assim, obtenção do resultado. $\mathrm{Ou}$ seja, é uma espécie de programação de textos a fim de que tenham um padrão específico de formatação, como a ABNT. Logo, essa ferramenta auxilia na escrita de equações, tabelas, citações e referências, por exemplo, sendo uma ferramenta leve, regular, sem falhas, gratuita e disponível em diversas versões e, até mesmo, online.

O Latex é utilizado em diversas disciplinas a fim de elaborar relatórios práticos, como em Práticas de Laboratório, Circuitos Elétricos, Eletrônica Básica, Laboratório de Controle Automático, Projeto Orientado, além de muitas outras disciplinas que não necessariamente são práticas, mas que exigem relatórios ou trabalhos acadêmicos. O conhecimento dessa ferramenta não é obrigatório, uma vez que existem outros programas para edição de texto no mercado, entretanto, alguns professores exigem que o documento seja feito exclusivamente em Latex. Apesar da não obrigatoriedade, essa ferramenta é muito útil e reduz o trabalho do aluno na edição e formatação de elementos como equações complexas. Nesses casos, a edição desse tipo de elemento em outros programas é mais trabalhosa que no Latex. Além das disciplinas do curso, essa ferramenta é muito utilizada na elaboração de relatórios e submissões de projetos em Iniciações Científicas e demais projetos de extensão. Também é muito utilizado na disciplina de Projeto de Graduação para a escrita do TCC devido à sua fácil adequação com a formatação exigida pela ABNT. 


\section{Matlab}

O Matlab é um software da MathWorks Inc. e sua aplicação mais geral se dá nos cálculos com matrizes. De maneira mais específica, é muito utilizado em simulações, resolução de equações, modelagem de sistemas; tudo isso com possibilidade de visualização dos resultados em gráficos de maneira fácil e clara. Com diversas bibliotecas já desenvolvidas, o software oferece funções que realizam cálculos e tarefas de maneira muito mais fácil quando se compara com a execução em linguagens de programação como $C$ ou Fortran. Essa ferramenta é aplicada em disciplinas que são cursadas principalmente da metade até o fim do curso, tanto obrigatórias quanto optativas, de todas as ênfases.

Em se tratando de disciplinas obrigatórias, é usado em Algoritmos Numéricos para a construção de códigos com objetivo de encontrar soluções numéricas para sistemas de equações não-lineares, para interpolação, integração numérica, entre outros. Em disciplinas da área de Controle e Automação, como Análise e Modelagem de Sistemas Dinâmicos e Sistemas Realimentados, é utilizada não apenas como coadjuvante, mas faz parte dos instrumentos avaliativos de tais disciplinas (em provas e trabalhos). É usado para modelagem e simulação de diversos sistemas de controle, sintonia de controladores PID, etc. Já em Princípio de Comunicações (área de Telecomunicações), é vastamente utilizado para fixação dos conteúdos apresentados (modulação, transmissão de sinais de voz e imagem, multiplexação/demultiplexação, entre outros), também sendo utilizado em suas atividades avaliativas.

Há uma disciplina prática, chamada Laboratório de Controle Automático, que se baseia totalmente na utilização de Matlab em conjunto com Arduino e Simulink para elaboração de experimentos relacionados a sistemas de controle.

Com relação às disciplinas optativas, novamente o Matlab é bastante utilizado em casos como: Transmissão de Energia Elétrica, Acionamentos Elétricos e Análise de Sistemas de Energia Elétrica.

\section{MINICURSOS OFERTADOS E ANÁLISE DOS DADOS}

\subsection{Contexto de Aplicação dos Minicursos}

Ao longo dos anos, o grupo PET Engenharia Elétrica Ufes, nas suas mais diversas composições de integrantes, realizou uma série de atividades envolvendo a graduação e a comunidade geral, das quais destacam-se os minicursos. A necessidade de domínio ou, ao menos, conhecimento de ferramentas de simulação, edição de textos, formulações matemáticas, manejo de circuitos eletrônicos, dentre outros, exigida no curso é incompatível com a abstenção da abordagem desses conteúdos na sala de aula.

Em meio a esse contexto, buscando ajudar os alunos e desenvolver atividades previstas no tripé acadêmico, os seguintes minicursos já foram ministrados pelo PET Elétrica: Excel, Matlab, Latex, Eagle, teoria de Grafos e Redes Complexas, além de microcontroladores, controladores e sistemas embarcados como Arduino, Raspberry PI, PIC e CLP (Controladores Lógico Programáveis). A maioria desses minicursos já foi ofertada, não somente para graduandos de Engenharia Elétrica da Ufes e de outras instituições de ensino superior, como também para outros cursos e pessoas da comunidade externa.

A Figura 1 apresenta fotos de duas turmas de minicurso ofertadas. Tendo em vista a recorrência da mesma problemática em outros cursos, sobretudo, os que apresentam pontos em comum no Projeto Pedagógico de Curso (PPC), é natural que ele seja aplicado para outros 
alunos. Contudo, a abrangência é ainda maior que a esperada quando é percebida a crescente demanda por novas tecnologias no panorama educacional e laboral atual, o que resulta na busca desses minicursos por estudantes de áreas que vão além da Engenharia, Matemática e Física.

a) Arduino

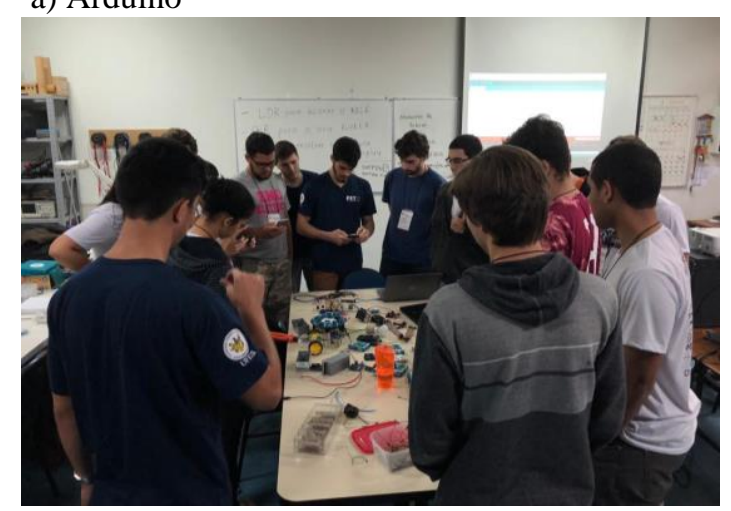

Figura 1 - Aplicação dos minicursos b)Latex

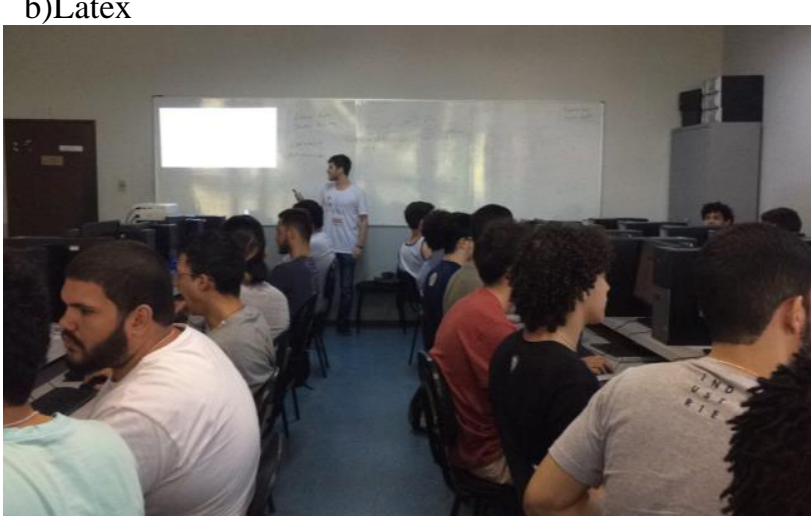

Fonte: Próprios autores.

A realização dessas atividades tendo como público a comunidade externa também é um ponto forte no grupo. Além das edições de minicursos esporádicas ou feitas com base numa demanda dos graduandos, o PET Elétrica já organizou minicursos em eventos como a JACEE (Jornada de Atualização em Computação, Elétrica e Eletrônica), um evento bienal realizado pelo PET Engenharia Elétrica em conjunto com o PET Engenharia da Computação, ambos da Ufes, e na SENG (Semana da Engenharia). Especialmente nessas edições, a diversidade de alunos envolvida foi particularmente singular, abarcando diferentes cursos, instituições de ensino e mesmo pessoas externas à esfera acadêmica, o que mostra um compromisso com a Extensão, um dos eixos do tripé acadêmico.

\subsection{Dados de Alcance do Projeto de Minicursos}

Conforme mencionado, muitos foram os minicursos e oficinas elaborados e ministrados pelos petianos. Como há carência de informação (como número de participantes, quantidade de minicursos e oficinas ministradas por ano, feedbacks dos participantes), os comentários e análises foram feitos baseados apenas nos principais minicursos que ocorreram entre os anos de 2017 a 2019, por possuírem dados mais recentes e um pouco mais detalhados. Apesar do esforço realizado pelo grupo, nos últimos anos, para manter um registro mais detalhado de suas atividades, ainda não foi possível obter uma base de dados que se possa dizer que seja a mais adequada para realizar comparações e análises mais complexas. Além disso, esse período corresponde ao da participação dos autores no programa.

A seguir, será feito um detalhamento dos minicursos oferecidos.

\section{Arduino}

Ganhando espaço nas disciplinas e projetos externos na atualidade, o minicurso de Arduino sempre foi requisitado pelos alunos. Em razão da necessidade do uso de sensores e componentes eletrônicos de posse do grupo, muitas vezes os alunos fizeram o minicurso em duplas. No ano de 2019, houve 42 inscritos, incluindo alunos da Ciência da Computação e da Engenharia Mecânica, além dos graduandos em Engenharia Elétrica. O minicurso tem sido 
abordado de forma mais prática, tendo pequenos projetos como base de aprendizado, o que foi avaliado positivamente pelos alunos nos feedbacks enviados ao grupo.

\section{Excel}

Iniciado em 2018, o minicurso de Excel atraiu a presença de alunos dos mais diversos cursos, como Engenharia Elétrica, Engenharia Mecânica, Administração, Engenharia Civil, Engenharia da Computação, Oceanografia, dentre outros. Esse minicurso foi dividido em dois módulos: Básico e Intermediário/Avançado. Inicialmente, no módulo Básico, eram abordadas as ferramentas básicas do Excel, além de algumas fórmulas mais utilizadas e comuns a diferentes contextos de aplicação. Bons feedbacks foram recebidos, sendo então formulado e aplicado o segundo módulo, Intermediário/Avançado, que procurou dar sequência ao conhecimento adquirido no módulo anterior, aprofundando um pouco mais em ferramentas e fórmulas mais complexas, adentrando ao final no conceito de Macros. Apesar da limitação encontrada referente à quantidade de computadores, a qual impactou no número de vagas disponíveis, os feedbacks de ambos os módulos foram elogiosos, sobretudo, no que que diz respeito à quantidade de exercícios produzidos.

\section{Latex}

O minicurso de Latex foi modificado ao longo de suas aplicações. Inicialmente fazendo uso da plataforma TEXStudio; e, a partir de 2018, utilizando a plataforma online Overleaf. No ano de 2017, foi utilizado como espaço o Laboratório de Computação da Elétrica e Eletrônica (LCEE). Já em 2018, o minicurso foi aplicado na IV JACEE e, em 2019, na sala do PET Elétrica. Em um feedback realizado em 2019, o minicurso foi bem elogiado, sobretudo a respeito da metodologia didática utilizada, pautada em exemplos. A quantidade de computadores muitas vezes limitou a quantidade de alunos participantes, bem como os horários das aulas, ainda assim, muitos foram beneficiados pela aplicação do minicurso.

\section{Matlab}

Ao longo de suas aplicações, o minicurso abordou, em geral, assuntos mais básicos (como a sintaxe adotada na programação, estruturas simples) e que podem ser aplicados em diversas funções. Com o passar do tempo e com aplicações cada vez mais específicas, em 2019, o minicurso foi remodelado e passou a oferecer também edições avançadas, quando tópicos como tratamento e reconhecimento de imagem e modelagem via Simulink começaram a ser abordados. Essa expansão no escopo resultou em um alcance muito grande de alunos, limitado somente pela disponibilidade de vagas. Os feedbacks mostraram a necessidade que os alunos sentiam de um minicurso que abordasse tais temas, com vários alunos reiterando que as aulas os ajudaram diretamente da execução de trabalhos.

No total, contando apenas esses quatro minicursos, houve a presença de 245 participantes nos três anos analisados. A título de comparação, no mês de junho de 2020 haviam 475 alunos matriculados no curso de Engenharia Elétrica da Ufes.

O Gráfico 1 apresenta a distribuição dos participantes por minicurso, onde é possível perceber que os quatro minicursos tiveram uma média de procura e interesse de participação semelhante. O Gráfico 2, por sua vez, mostra a quantidade de alunos participantes em cada minicurso, em cada um dos três anos analisados. Nele, é possível observar uma demanda crescente de participação a cada ano, o que reforça a importância desses minicursos na realização das disciplinas do curso de engenharia, uma vez que, a cada nova edição, os alunos 


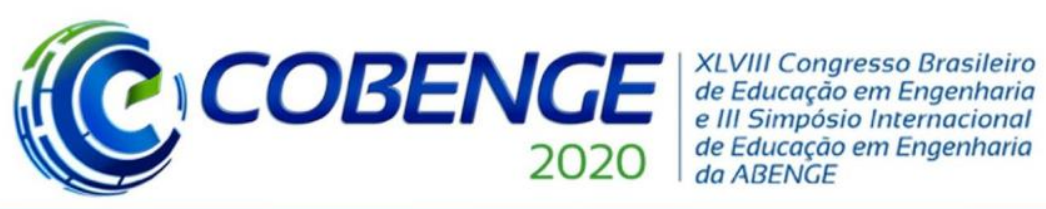

"Os desafios para formar hoje o engenheiro do amanhã"
$\mathrm{Ol} \mathrm{a} \mathrm{O3}$ de dezembro Evento On-line

que já participaram acabam fazendo propaganda do minicurso para os outros alunos, fazendo com que sua demanda seja ainda maior.

Gráfico 1 - Distribuição de participantes por assunto

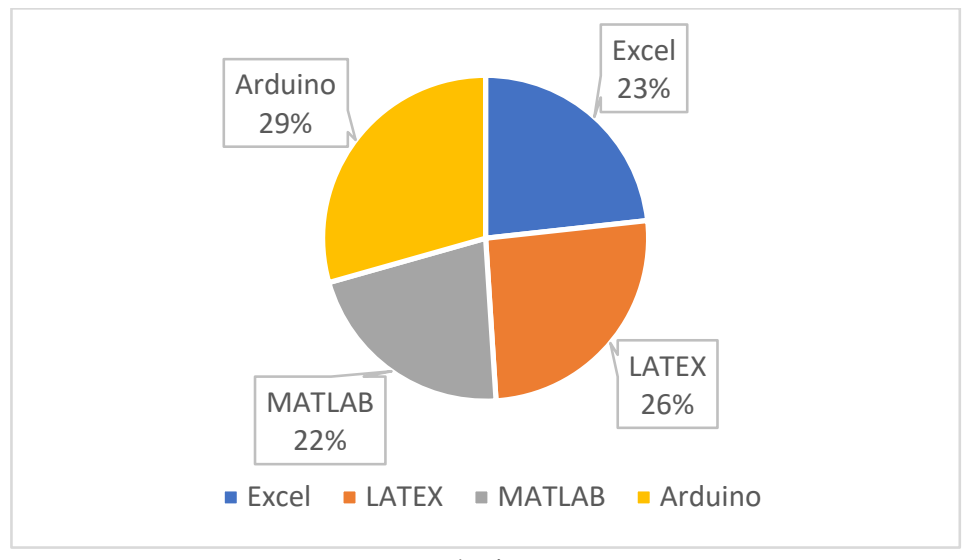

Fonte: Próprios autores

Gráfico 2 -Participantes por minicurso

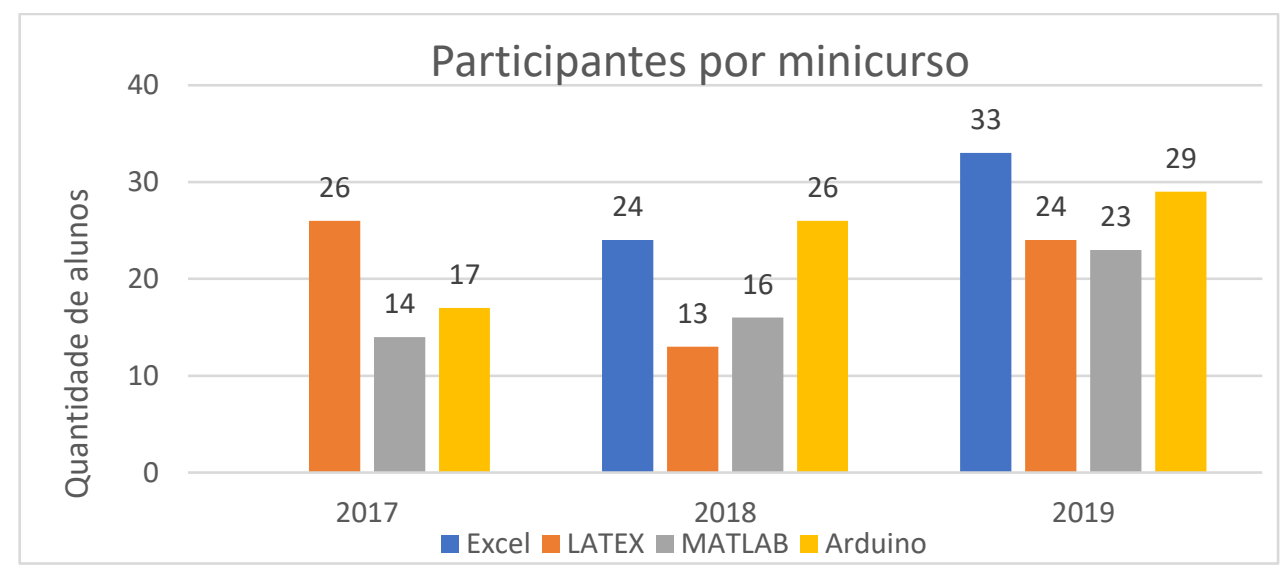

Fonte: Próprios autores

\subsection{Confecção de Placas de Circuito Impresso}

O PET Engenharia Elétrica contribui, ainda, na formação de conhecimento a respeito da confecção de placas de circuito impresso. Como parte da disciplina de Práticas de Laboratório, o grupo habilita os alunos já no primeiro período a desenvolverem placas que são utilizadas não apenas como projeto da disciplina, mas também em diversos trabalhos ao longo de todo o curso, nas cadeiras de Eletromagnetismo, Circuitos Elétricos, e, muitas vezes, na elaboração de seu TCC na disciplina Projeto de Graduação. Nessa aula, são abordados os aspectos de software (por meio da ferramenta Eagle, da Autodesk) e os de hardware (impressão do circuito, transferência para a placa, corrosão, soldagem).

Não obstante, essa também é uma oportunidade importante do grupo PET estabelecer contato com os ingressantes do curso de Engenharia Elétrica, uma vez que a disciplina na qual esta aula acontece é obrigatória. 


\section{SOBRE AS HABILIDADES E BENEFÍCIOS OBTIDOS ATRAVÉS DOS MINICURSOS}

Durante a estruturação do minicurso, existem algumas etapas que são realizadas antes do minicurso estar realmente pronto para aplicação à graduação. Por meio da idealização, os petianos conseguem passar por momentos de brainstorming com o grupo, obtendo melhores métricas para o desenvolvimento da ementa e material do curso. Após esse processo, existe uma grande preparação por parte daqueles que se tornarão os professores durante a realização das aulas, o que os leva a adquirirem um conhecimento mais aprofundado sobre as diferentes ferramentas que se apresentam como tema. Habilidades interpessoais, técnicas e metodologias didáticas também são desenvolvidas nesses alunos.

Para aqueles que, por outro lado, participam do minicurso, obtém-se um aprendizado fora da sala de aula tradicional do curso, e a possibilidade de aprimoramento técnico, por meio da participação de aulas, as quais são conduzidas por outros colegas, com uma linguagem acessível e materiais bem estruturados, tornando a assimilação de conhecimento muito maior.

O conhecimento sobre ferramentas vastamente utilizadas no meio industrial e acadêmico, traz uma gama de oportunidades aos alunos envolvidos. O graduando se aproxima um pouco mais dos conhecimentos técnicos mais buscados nesse meio, os quais não são encontrados na sala de aula tradicional. A qualidade e excelência na formação acadêmica é elevada, tornando o aluno mais preparado e facilitando seu acesso ao mercado de trabalho.

\section{CONSIDERAÇÕES FINAIS}

Com o passar dos anos, tem-se visto um mercado cada dia mais competitivo, exigindo cada dia mais do profissional e de seu aperfeiçoamento e capacitação. No intuito de responder a essa necessidade e capacitar melhor os alunos de engenharia elétrica da Ufes, os minicursos ofertados pelo PET Elétrica tem representado um papel fundamental tanto na formação dos alunos de graduação quanto na dos petianos envolvidos, atuando como uma complementação à sua formação acadêmica e elevando a qualidade do profissional que é formado no curso de Engenharia Elétrica da Ufes. Nesse sentido, também se verifica que o grupo PET tem participação ativa na trajetória acadêmica de grande parte dos alunos, seja por meio da aula de confecção de placas, dos minicursos ou das diversas outras atividades de ensino, pesquisa e extensão realizadas por ele.

\section{REFERÊNCIAS}

BRASIL. Portaria n . 976 de 27 de julho de 2010. Dispõe sobre o Programa de Educação Tutorial - PET. Diário Oficial [da] República Federativa do Brasil, Poder Executivo, Brasília, DF, 27 jul. 2010. Seção 1, p. 40.

BRASIL. Portaria n . 343 de 24 de abril de 2013. Diário Oficial [da] República Federativa do Brasil, Poder Executivo, Brasília, DF, 24 abr. 2013. Seção 1, p. 24. 
"Os desafios para formar hoje o engenheiro do amanhã"

Ministério da Educação - MEC, 2020. Cadastro Nacional de Cursos e Instituições de Educação Superior. Disponível em http://emec.mec.gov.br/. Acesso em: 08 de julho de 2020.

Coordenação de Aperfeiçoamento de Pessoal de Nível Superior - CAPES. Plataforma Sucupira. Disponível em https://sucupira.capes.gov.br/sucupira/public/consultas/coleta/programa/viewPrograma.xhtml ?popup=true\&cd_programa=30001013004P0. Acesso em: 23 de Junho de 2020.

Departamento de Engenharia Elétrica Ufes - DEE/UFES. Engenharia Elétrica. Disponível em: http://www.engenhariaeletrica.ufes.br/pt-br. Acesso em 22 de junho de 2020.

Folha de S. Paulo. Ranking de Cursos de Graduação. Disponível em https://ruf.folha.uol.com.br/2019/ranking-de-cursos/engenharia-eletrica/. Acesso em: 23 de junho de 2020.

Ministério da Educação - MEC. Apresentação - PET. Disponível em: http://portal.mec.gov.br/pet. Acesso em: 09 de junho de 2020.

PET Ufes. O Programa de Educação Tutorial. Disponível em: http://pet.ufes.br/. Acesso em: 08 junho de 2020.

Universidade Federal do Espírito Santo - UFES. Currículo de Cursos: Engenharia Elétrica. Disponível em:

http://www.engenhariaeletrica.ufes.br/sites/engenhariaeletrica.ufes.br/files/field/anexo/ppc 22.05.2018.pdf. Acesso em: 24 de junho de 2020.

\section{THE ROLE OF THE MINICOURSES IN TRAINING FOR THE USE OF ESSENTIAL TOOLS TO THE COURSE OF ELECTRICAL ENGINEERING: EXPERIENCE OF PET ELECTRICAL UFES}

Abstract: Electrical Engineering is a wide area of knowledge, not being possible the exploration, in the graduation courses, of all of its fields of action and necessary tools to the comprehension of those. The minicourses offered by the PET Electrical Engineering UFES are seen, before this scenario, as an alternative for the complementation of what is exposed in the classroom, as well as the exigences of the labor market. It was shown, therefore, through means of data of participation and feedbacks of the minicourses which were applied, besides the reports of the authors (former participants of the group), that the impact of this activity is beneficial to the students as well as to the course itself. It is also detailed that the development of activities of this kind (teaching minicourses) is in perfect alignment with the principles of the PET.

Keywords: Minicourses. Electrical Engineering. PET Electrical Engineering Ufes. 